\title{
Discriminating thermodynamic and kinetic contributions to rare earth element uptake in garnet
}

\author{
MATTHIAS KONRAD-SCHMOLKE ${ }^{1}$, RALF HALAMA ${ }^{2}$ \\ AND DAVID M CHEW ${ }^{3}$ \\ ${ }^{1}$ Earth Science Department, University of Gothenburg \\ ${ }^{2}$ Keele University \\ ${ }^{3}$ Trinity College Dublin \\ Presenting Author: mks@gvc.gu.se
}

Rare earth element (REE) patterns in metamorphic garnet are an important source of information of geodynamic and geochemical processes. In order to extract this information, the thermodynamic and kinetic contributions of the REE uptake in garnet must be distinguished and quantified. Utilizing high resolution trace element and $\mu$-Raman mappings together with combined thermodynamic-geochemical-diffusion models we demonstrate that equilibrium and kinetic aspects of the REE uptake in metamorphic garnet can be discriminated by interpreting $2 \mathrm{D}$ trace element mappings in a single sample. The heavy REE ( $\mathrm{Tb}$ to $\mathrm{Lu}$ ) zoning in the investigated garnet comprises an inner part with a bell-shaped pattern, but decreasing height of the central peak with decreasing atomic number of the REE and a concentric zone of HREE enrichment followed by drastic HREE decrease towards the rim. The broad overall shape of this pattern resembles those often observed in metamorphic garnets from different rock types and tectonic settings. Superimposed on this trend is a concentric pattern of minor recurring fluctuations in the REE concentrations with at least six regularly spaced sets of peaks and troughs along the entire garnet radius. Comparison of the observed inclusion suite, the trace element maps and thermodynamic-geochemical models show that the inner bell-shaped part results from fractional garnet growth in an unchanged mineral assemblage, whereas the REE enrichment zone is caused by the breakdown of titanite. The width of the central peak is controlled by the bulk permeability of the interconnected transport matrix and the fraction of matrix minerals the garnet equilibrates with. The superimposed REE fluctuations result from changing element transport properties of the host rock and mark recurring changes from equilibrium REE uptake to transport-limited REE uptake in garnet. Such fluctuating element transport properties can be best explained by pulse-like fluid fluxes that rhythmically change the interconnectivity of the intercrystalline transport matrix. 